\title{
Improvement of EDM Machining Speed by Using Magnetic/Piezoelectric Hybrid Drive Actuator
}

\author{
Xiaoyou Zhang and Kouki Uchiyama \\ Department of Mechanical Engineering, Nippon Institute of Technology, 4-1, Gakuendai, Miyashiro-cho, Minamisaitama-gun, Saitama, \\ Japan
}

\begin{abstract}
The objective of this research is to achieve high-speed electrical discharge machining (EDM) of small holes using a high-speed, high-precision, 3-DOF controlled, magnetic/piezoelectric hybrid drive actuator. In this paper, the proposed actuator was attached to the conventional electrical discharge machine, and the increase in the machining speed using the actuator was experimentally confirmed. The relationships between the machining speed, the amplitude and the frequency of the electrode vibration were discussed. Experimental results show that the use of the proposed actuator can speedily adjust the distance between the electrode and the workpiece, and the machining speed was increased by $138 \%$ compared with the conventional EDM. Moreover, the machining speed using the actuator also was increased as the changes of the amplitude and the frequency of the electrode vibration, and it was increased by $76 \%$ compared to without the electrode vibration.
\end{abstract}

\section{Introduction}

Electrical discharge machining (EDM) is a nonconventional material removal processes based on the thermo-electric energy created between an electrically conductive workpiece and an electrode submerged in a machining fluid. EDM has several advantages such as the capability of machining of all conductive materials, regardless of their hardness, and the ability to deal with complex shapes[1]. However, the machining speed and accuracy of conventional EDM are limited by probability and efficiency of the electrical discharges. To obtain a stable electrical discharge, the electrode needs to be speedily re-positioned in order to maintain a suitable distance from the workpiece, and the debris around the electrode due to EDM has to be removed immediately.

To improve the positioning response and the positioning accuracy, a combination of a conventional electrical discharge machine and a wide-bandwidth, highprecision, multi-degree- of-freedom local actuator is required. As the local actuators, a piezoelectric actuator[2], a voice coil motor[3], and a magnetic bearing[4] have been used to improve the positioning response of the electrode.

Moreover, to immediately remove the debris around the electrode, in the conventional EDM, the cylindrical electrode is usually rotated or jumped. The fresh machining fluid can be introduced into the machined hole and the debris can be removed. However, in the rectangular hole machining or 3D surface machining, the electrode cannot be rotated, and adhesion of the workpiece and electrode due to the improper flow of the debris is a problem. To increase the flushing effect during the EDM, an ultrasonic vibration has been superposed on the normal electrode movement[5]. Furthermore, to flush out debris from the machined hole, a vibration-assisted machining also has been introduced to micro-EDM using piezoelectric[6]

In this paper, the developed 3-DOF controlled, highspeed, high-precision, local actuator is attached to an electrical discharge machine to experimentally confirm the increase in the machining speed. Then, the relationships between the machining speed, the amplitude and the frequency of the electrode vibration are discussed.

\section{Magnetic/piezoelectric hybrid drive actuator}

Fig. 1 shows the principle of the proposed 3-DOF controlled, magnetic/piezoelectric hybrid drive actuator. The actuator primarily consists of a thrust magnetic bearing, a pair of elastic hinges, and an electrode vibration mechanism in the radial directions. The motion of the spindle attached the electrode in the $\mathrm{Z}$ direction is controlled by the voice coil motor type of thrust magnetic bearing consisting of an air-core coil and two permanent magnet rings, as shown in Fig. 1(a). The thrust magnetic bearing with a positioning stroke of a few millimeters can realize speedy position of the electrode in order to maintain a suitable distance from the workpiece, and the fast jump theelectrode to introduce fresh machining fluid into the hole and wash away debris. The motions of the spindle in $\mathrm{X}, \mathrm{Y}, \Theta, \Phi$ and $\Psi$ directions are constrained by 
upper and lower elastic hinges shown in Fig. 1(b).

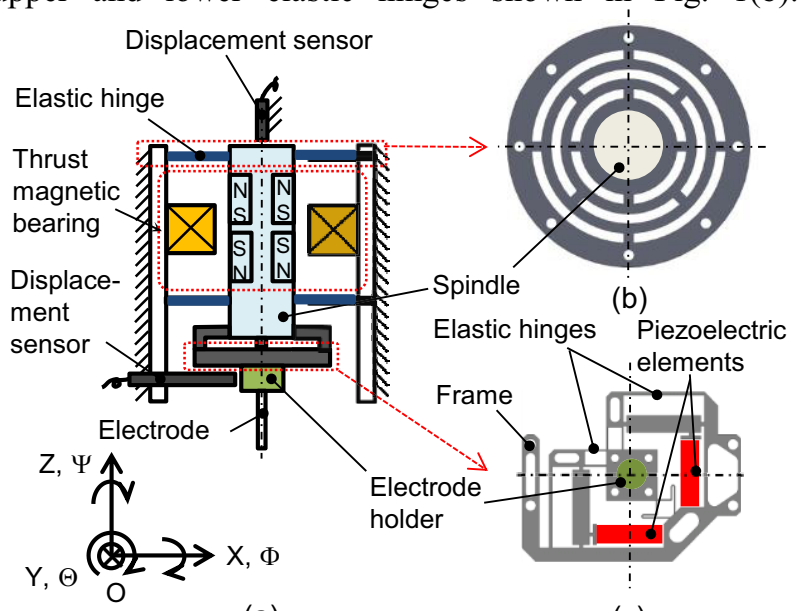

(a)

(c)

Figure 1. Configuration of 3-DOF controlled actuator

The electrode vibration mechanism shown in Fig. 1(c) is attached to the lower end of the spindle. The main components comprise elastic hinges, two piezoelectric elements, and an electrode holder. The electrode used to machining is fixed by the electrode holder. The motions of the electrode in $\mathrm{X}$ and $\mathrm{Y}$ directions are driven by two piezoelectric elements, to achieve the vibration of the electrode to remove the debris. To realize a requested actuation stroke at higher operating frequencies, a displacement magnification mechanism for piezoelectric elements are applied.

The height of the actuator is $97 \mathrm{~mm}$, the diameter is $110 \mathrm{~mm}$, and the mass is $4.7 \mathrm{~kg}$. The actuator possesses a positioning resolution of $1 \mathrm{~m}$, a bandwidth of $120 \mathrm{~Hz}$ and a positioning stroke of $2 \mathrm{~mm}$ in the thrust direction, and a positioning resolution of $0.05 \mathrm{~m}$, a positioning stroke of $20 \mathrm{~m}$ at $1000 \mathrm{~Hz}$ in the radial directions.

\section{Electrical discharge machining system using the local actuator}

In order to improve the machining speed of EDM, the developed actuator was attached to an electrical discharge machine (EA8PV, Mitsubishi Electric Corp.), as shown in Fig. 2. In the experiments, the conventional electrical discharge machine was used only to move the electrode to the initial position and to supply the voltage to the electrode. The electrode was controlled solely by the local actuator.

A cylindrical shaped copper electrode with a diameter of $2 \mathrm{~mm}$ was used and was not rotated or retracted during EDM. The machining fluid was EDF-K (Nippon Oil Corp.). The EDM experiments were carried out, until a $0.5 \mathrm{~mm}$ thick SUS304 steel plate was penetrated. The discharge circuit was a transistor circuit with a peak current of $29.0 \mathrm{~A}$, a pulse on-time of $70.8 \mu \mathrm{sec}$, and an offtime of $115.2 \mu \mathrm{sec}$.

To maintain a stable electrical discharge, the voltage between the electrode and the workpiece was kept at a constant value by rapidly changing the distance between the electrode and the workpiece. A block diagram of the EDM system is shown in Fig. 3. The gap voltage $V$, is

amplified and averaged by a low-pass filter, and the average voltage, $V_{f b}$, is used as a feedback signal. The gain of the amplifier is 0.03 and the cutoff frequency of the low-pass filter is $330 \mathrm{~Hz}$. The deviation between the reference voltage, $V_{r}$, and the feedback voltage, $V_{f b}$, is sent to a PI controller to calculate the value $V_{a r}$, which is used to position the electrode with respect to the workpiece. The reference $\boldsymbol{R}$ is the motion path of the electrode. To give a stable electrical discharge, the reference voltage $V_{r}$, was set to $1.6 \mathrm{~V}$, and the values of and in the PI controller were set to $1.0 \times 10^{-6}$ and $1.0 \times 10^{-4}$, respectively, by trial and error.

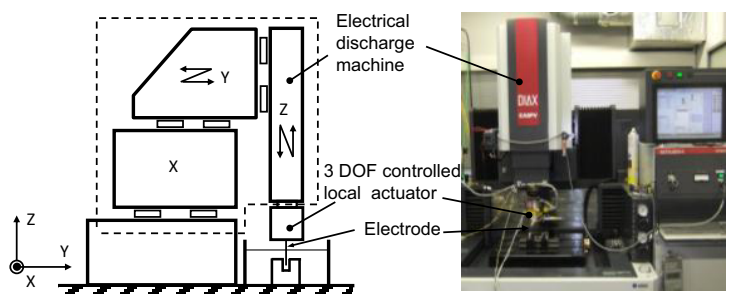

Figure 2. Electrical discharge machine with local actuator

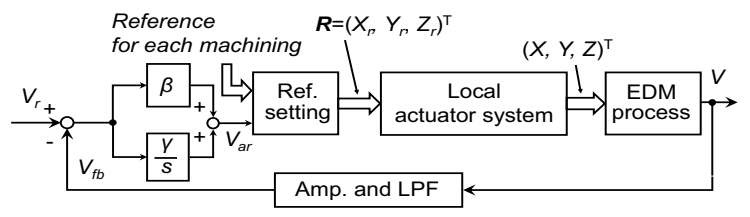

Figure 3. Block diagram of EDM system

\section{Electrical discharge machining experiments}

\subsection{Hole machining without electrode vibration}

\subsubsection{EMD method}

In order to evaluate the effectiveness of the proposed actuator for improvement of EDM machining speed, straight holes were machined in the thrust direction. In EDM experiments, the electrode position in this direction is controlled by the voltage across the gap between the electrode and the workpiece, and the electrode vibration in the radial directions is not carried out. In this case the $Z_{r}$ of the input reference $\boldsymbol{R}$ of the actuator system was set to $V_{a r}$, and $X_{r}, Y_{r}$ were set to zero. That is,

$$
R=\left(\begin{array}{lll}
X_{r} & Y_{r} Z_{r}
\end{array}\right)^{T}=\left(\begin{array}{lll}
0 & 0 & V_{a r}
\end{array}\right)^{T}
$$

For comparison, an EDM experiment was also carried out using the conventional electrical discharge machine only. The parameters of the PI controller and the machining conditions were identical to those used for the actuator.

\subsubsection{Machining results}

Fig. 4 shows photographs of the machined holes without and with the proposed actuator. Using the proposed 
actuator, the diameter of the hole was $2.13 \mathrm{~mm}$. On the other hand, using conventional EDM only, the diameter of the hole was $2.14 \mathrm{~mm}$, and the diameters were almost the same. The feed displacements of the electrode in the thrust direction were measured to evaluate the motion of the electrode during EDM as shown in Fig. 5. By using the proposed actuator, the machining time was reduced from $124.8 \mathrm{~s}$ to $50.8 \mathrm{~s}$.

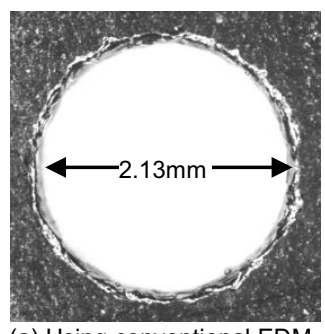

(a) Using conventional EDM

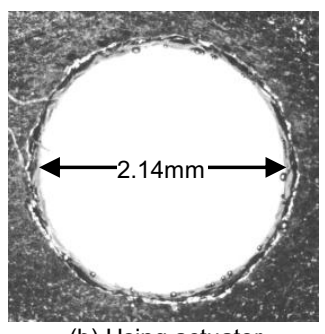

(b) Using actuator
Figure 4. Machined holes

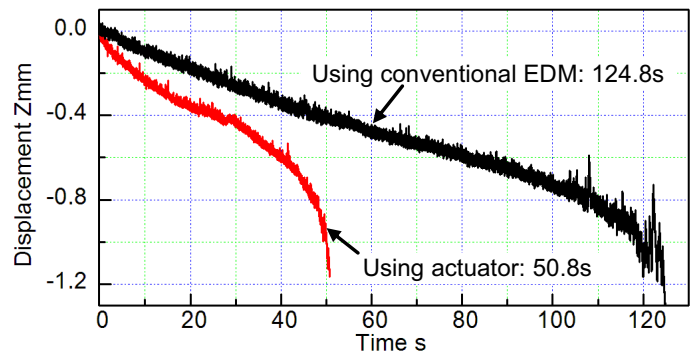

Figure 5. Feed displacements of electrode

Moreover, the feedback voltage $V_{f b}$, was monitored by a digital signal processor (DS1103, dSPACE Corp., Resolution, 16bit) at a sampling frequency of $10 \mathrm{kHz}$, as shown in Fig. 6. Comparing Fig. 6(a) with Fig. 6(b), it can be seen that the response of the electrode to maintain a stable electrical discharge is enhanced when using the proposed actuator.

For each EDM experiment, several holes were machined under the same conditions. Five holes except the holes influenced by large abnormal electrical discharges were used for the sample data. Defining the average machining speed as the ratio of thick of the workpiece to averaged machining time, the average machining speeds using the maglev local actuator and using only a conventional EDM were $9.59 \mathrm{~m} / \mathrm{s}$ and $4.03 \mathrm{~m} / \mathrm{s}$, respectively. Thus, the average machining speed was improved by $138 \%$.

\subsection{Hole machining with electrode vibration}

\subsubsection{EMD method}

To evaluate the relationships between the machining speed, the amplitude and the frequency of the electrode vibration, the amplitude and the frequency of the electrode in radial directions were tuned respectively during the EDM experiments. In this case, the electrode is drived in a circular path in the radial directions, and $\boldsymbol{R}$ is given by Eq. (2).

$$
R=\left(X_{r} Y_{r} Z_{r}\right)^{T}=\left(\frac{A}{2} \cos 2 \pi f t \frac{A}{2} \sin 2 \pi f t V_{a r}\right)^{T}
$$
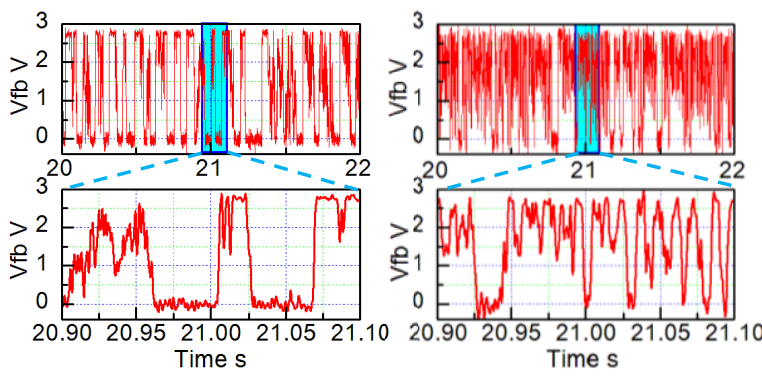

(a) Using conventional EDM

(b) Using actuator

Figure 6. Feedback voltage $\left(V_{f b}\right)$ during EDM

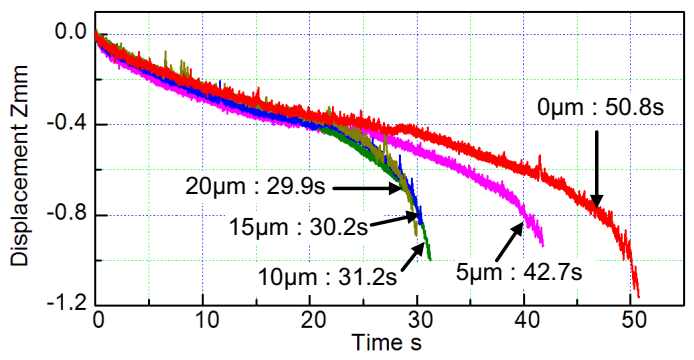

Figure 7. Feed displacements of electrode on $1000 \mathrm{~Hz}$

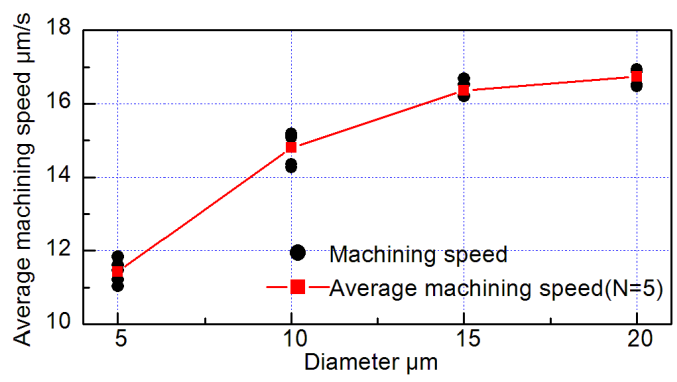

Figure 8. Average machining speed on $1000 \mathrm{~Hz}$

Where $A$ and $f$ are the diameter and frequency of the circular path, respectively. The frequency of the circular path was set to $200 \mathrm{~Hz} \sim 1200 \mathrm{~Hz}$, and the diameter was set variously to $5 \mu \mathrm{m} \sim 20 \mu \mathrm{m}$.

\subsubsection{Machining results}

Firstly, the frequency of the circular path was set to $1000 \mathrm{~Hz}$, and the diameter was tuned to $5 \mathrm{~m}, 10 \mathrm{~m}$, $15 \mathrm{~m}, 20 \mathrm{~m}$ in EDM experiments. Fig. 7 shows the feed displacements of the electrode in the thrust direction. From these results, it can be seen that as the amplitude of the electrode vibration was increased, the machining time can be reduced to a saturated value.

For each EDM experiment, Five holes were also machined under the same conditions. The relationships between average machining speed and the amplitude of the electrode vibration are shown in Fig. 8. As the amplitude of the electrode vibration was increased, the average machining speed also increased. Therefore, to realize high speed machining, it is necessary to select a large the amplitude for the electrode vibration. However, for the accurate hole machining, it is necessary to 
consider the influence of the amplitude of the electrode vibration.

Secondly, the diameter of the circular path was set to $15 \mu \mathrm{m}$, and the frequency was tuned to $200 \mathrm{~Hz}, 350 \mathrm{~Hz}$, $600 \mathrm{~Hz}, 800 \mathrm{~Hz}, 1000 \mathrm{~Hz}$ and $1200 \mathrm{~Hz}$. Five holes were also machined under the same conditions, and the calculated average machining speed is shown in Fig. 9. When the frequency of the electrode vibration is smaller than $1000 \mathrm{~Hz}$, the average machining speed gradually increases.

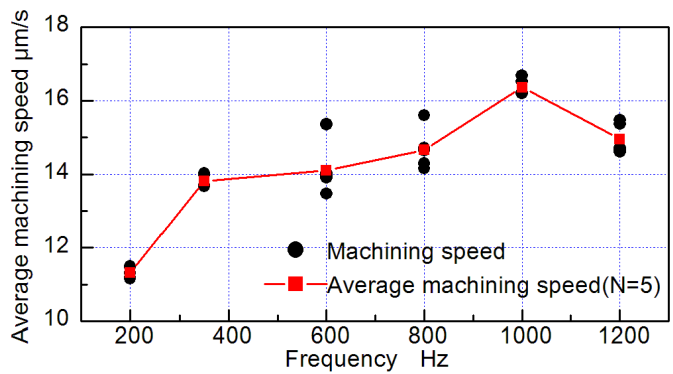

Figure 9. Average machining speed on $15 \mu \mathrm{m}$

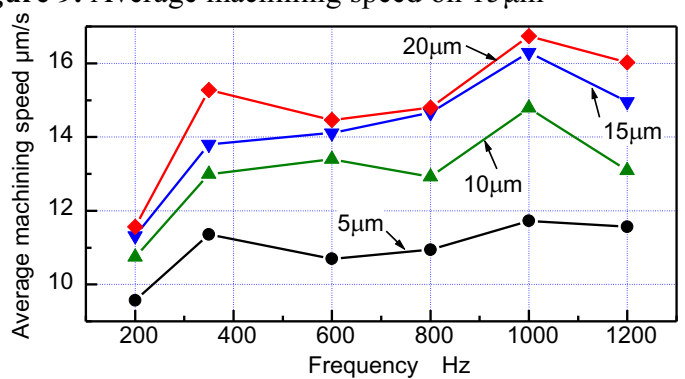

Figure 10. Relationships between average machining speed, amplitude and frequency of electrode vibration

The relationships between the average machining speed, the amplitude and the frequency of the electrode vibration are shown in Fig. 10. When the diameter of the circular path was set to $20 \mathrm{~m}$, and the frequency was set to $1000 \mathrm{~Hz}$, the machining speed becomes the maximun, and it was $16.7 \mathrm{~m} / \mathrm{s}$. The average machining speed was increased by $76 \%$ compared to without the electrode vibration, and $315 \%$ compared to conventional EDM.

\section{Conclusions}

In this paper, to evaluate the effectiveness of the proposed actuator for improvement of EDM machining speed, straight holes were machined in the thrust direction by using the proposed actuator. Experimental results show that the use of the proposed actuator can speedily adjust the distance between the electrode and the workpiece, and the machining speed was increased by $138 \%$ compared with the conventional EDM.

Moreover, the relationships between the machining speed, the amplitude and the frequency of the electrode vibration were discussed. The machining speed using the actuator also was increased as the changes of the amplitude and the frequency of the electrode vibration, and it was increased by $76 \%$ compared to without the electrode vibration, and $315 \%$ compared to conventional EDM.

\section{Acknowledgement}

This study was supported in part by JSPS KAKENHI Grant Number 15K05768 and SUZUKI Foundation.

\section{References}

1. T. Masuzawa: State of Art of Micromachining. Annals of the CIRP, Vol. 49 (2000), No.2, p. 473.

2. Y. Imai, A. Satake, A. Taneda and K. Kobayashi: Improvement of EDM Machining Speed by Using High Frequency Response Actuator. Int. J. Electr. Mach., (1996), No.1, p. 21.

3. T. Masuzawa, K. Tanaka and M. Fujino: Study on the High Speed Machining by EDM Using a Moving Coil Head Type Feed Control. In: Proceedings of the 19th International MTDR Conference, (1978), p. 543.

4. X. Zhang, T. Shinshi, T. Fukuoka and T. Nakai: Development of a 2-DOF Controlled Magnetic Drive Actuator for Laser Beam Cutting, Journal of Advanced Mechanical Design, Systems, and Manufacturing, JSME, Vol. 7 (2013), No. 3, p. 448.

5. H. Huang, H. Zhang, L. Zhou and H. Y. Zheng: Ultrasonic Vibration Assisted Electro-discharge Machining of Microholes in Nitinol. Journal of Micromechanics and Microengineering, Vol. 13 (2003), No.5, p. 693.

6. T. Endoa, T. Tsujimoto and K. Mitsui: Study of Vibration-assisted Micro-EDM. -The Effect of Vibration on Machining Time and Stability of Discharge. Precision Engineering, Vol. 32 (2008), No.4, p. 269. 